\title{
Staff perceptions and changing role of pre-hospital profession in the UK ambulance services: an exploratory study
}

\section{Introduction and background}

Management research on ambulance service is an emerging phenomenon (Wankhade and Mackway-Jones, 2015; Andrews and Wankhade, 2015; McCann et al., 2013; Wankhade, 2011, 2012, Heath and Radcliffe, 2007, 2010) notwithstanding some excellent ethnographic studies (Metz, 1981; Palmer, 1983; Tangherlini, 2000; Reynolds, 2009). This paper analyses the changing nature and scope of the ambulance work in the UK ambulance services, the coping behaviour of the ambulance staff and the resultant consequences of such intense work patterns for the organisation, individuals and society in general. The paper provides interesting insights about the work intensity of ambulance personnel in highlighting cultural and management challenges in coping with the rising demand in ambulance demand against the growing advances in clinical and educational role of the ambulance staff. Drawing from ethnographic case study data collected through interviews, observation and ambulance 'ride-ons', the paper will address the key issues in an English National Health Service (NHS) ambulance trust setting. Need for such an ethnographic exploration finds support in the literature (Anteby, 2013; Watson, 2011; Van Maanen, 2011; Mannon, 1992; Skolnick, 2008).

The ambulance service is the first point of access for a wide variety of health problems. When the NHS was created in 1948, the ambulance service was a function given to the local authorities. Unlike the Police and Fire Services, there was no accompanying legislation to provide structures and operational arrangements for the new ambulance services that were classified as 'essential' rather than 'emergency' services (Ambulance Service Association, 
2000, p. 7). Uniforms and rank structures emulated the established emergency services and a basic first aid certificate was all that was required to run the services. During the 1990s, ambulance services became NHS trusts. Being part of NHS since 1974, ambulance services have made huge progress towards its transformation from a patient transport organisation to a clinically trained workforce (National Audit Office NAO, 2010).

Academic literature on work intensity in a wide range of healthcare settings is gaining popularity (Klein et al., 2006; Gascoigne et al., 2015). The Keogh Urgent and Emergency Care Review (NHS England, 2013) highlighted the role of the ambulance services in the urgent and emergency care networks and their contribution towards reducing pressures in emergency departments (ED) is being acknowledged in various policy documents (NHS Five Year Forward View, 2014; NHS Confederation, 2014, 2015). Despite the efforts made by ambulance trusts to culturally transform themselves into a clinically driven workforce, they are still perceived as a quick response service dealing with life threatening emergencies. Lack of clinical educational and training of the ambulance workforce is still seen as a big cultural challenge (O’Mara et al., 2015; McCann et al., 2013; Price, 2006; Wankhade, 2011a, Wankhade and Brinkman, 2011; Siriwardena, 2010; Snooks et al., 2009). Vestiges of the old command and control culture, accompanied by tendency to blame, hierarchical and top-down management style, and resistance to change and being risk-averse (NHS Modernisation Agency, 2004) are some of the factors cited historically within ambulance service as barriers to transform from a 'blue collar trade' into a professional workforce (McCann et al., 2013). Ambulance leaders (Association of Ambulance Chief Executives AACE, 2011) have continued to argue about the need to professionalise the work force by transforming the work culture. Attempts on the part of the ambulance services to fully integrate within the wider NHS have 
also been hindered by the confusion which still prevails within its members about the core values and mission of the service (McCann et al., 2015; NHS Confederation, 2011).

This article examines the challenges and differing staff perceptions of the changing scope and practice of ambulance personnel. This line of enquiry offers interesting insights since the role of the ambulance worker is often expanded in public's mind as health professionals, but that the actual resources extended to front line staff do not match those expectations (such as lack of training, target chasing, long hours, no support, etc.). Meaningful insights are gained from staff perceptions about the nature of the work by the ambulance personnel in highlighting the cultural and management differences in being seen as an emergency service wearing uniforms and driving with lights and sirens (fast response) against the emerging role and expectation of being a clinically proficient pre-hospital care provider.

The article is structured as follows. Section one provides analysis of the changing scope and practice of ambulance paramedics in the UK and implications for patient safety. Section two reviews the literature on work intensity and its implications for staff sickness. Section three details the research methodology adopted in this study. Section four presents the study findings. This is followed by discussion and concluding remarks.

\section{Changing scope of ambulance practice and implications for patient safety}

Developing a professional workforce has been seen to be integral element of the ambulance modernisation programme and has been the focus of policy attention (NHS England, 2013; NAO, 2010; AACE, 2011; Department of Health DH, 2005). Historically, entry into the paramedic profession was attained in a technician role by working through various roles within 
a specific NHS ambulance trust in the UK. Following a period of experience, candidates would sit entry exams and then complete a training course to become a qualified paramedic. But this is no longer the case since 2008 and the recommended route to becoming a paramedic is now the completion of a higher education diploma in paramedic science or equivalent but with local and regional variations. Woollard (2009) while presenting details of different paramedic training initiatives around the UK, highlighted considerable variations in the 'length, content and academic level of the associated educational programmes. The Paramedics Evidence Based Education Project (PEEP) study commissioned by the DH (England), National Allied Health Professional Advisory Board and funded by the College of Paramedics, also highlighted great variations in the current education and training models which were very locally determined and resulted in very different student experiences and different levels of learning outcomes achieved at the point of registration (Lovegrove and Davis, 2013, p. 7). The study (Ibid, p. 9) recommended a standardised approach to all aspects of education and paramedic training and urged the College of Paramedics in partnership with National Education Lead Bodies to take the paramedic profession to an all graduate status by 2019 .

The title of 'Paramedic' is protected by law by the Health and Social Work Professions Order, 2001 and the paramedics are required to register with the regulatory body, the Health and Care Professions Council (HCPC). The College of Paramedics as the professional body for paramedics in the UK publishes the paramedic curriculum and competence framework (College of Paramedics, 2015, 2008). In all publications of the curriculum, the College stipulates the following paramedic roles namely, Student Paramedic; Paramedic; Specialist Paramedic; Advanced Paramedic and Consultant Paramedic all of which adhere and relate to the Skills for Health Career Framework. While there has been considerable discussion and movement towards the College structure a lack of holistic picture in the UK and 'national 
variation in the implementation of specialist paramedic roles was cited in the study by O'Hara et al., (2015, p.50) as one of the frustrations for the paramedics which was seen to be limiting career progressions and ability to fully utilize skills in ensuring they are dispatched to suitable patients.

It is however estimated that less than ten percent of emergency 999 callers having a life threatening condition (Evans et al., 2014). This has necessitated the need for providing alternate care options to a majority of the ambulance service users (Darzi, 2008). There is now a range of different specialist paramedic roles in the ambulance services including paramedic practitioners $(\mathrm{PP})$, emergency care practitioners $(\mathrm{ECP})$, critical care practitioners $(\mathrm{CCP})$ and community paramedics (CP) who are equipped with enhanced knowledge and skills needed to make complex decisions about patient care. It has been suggested that these specialist roles have reduced the conveyance of patients to EDs thus reducing the costs associated with ambulance journeys and increased discharge at scene (Hills et al., 2014). But current evidence has also raised concerns about safety of patents involved in non-conveyance decisions and the complexity of decision-making by the paramedics for people left at scene (O'Hara et al., 2015, p. 46). A recent scoping review (see Fisher et al., 2015 for more details) has illustrated the lack of quality information available regarding ambulance service patient safety in the UK. This study identified "many reports of cases for which operational pressures, including targets, were perceived to be more important than patient safety for ambulance services" and the need to understand the culture of the organisation with "consideration being given to the influence this can have on patient safety." (Ibid, p. iv-v). In another study, Halter et al., (2011) identified concerns about assessment and referral of older people with a fall who were left behind at the scene. In their study, Tohira et al., (2013) also pointed out the lack of rigorous evidence on the safety of patients while making decisions about treating patients at the scene. Current evidence 
concerning patient safety, effectiveness and funds to support these changes is arguably insufficient (Porter et al., 2007) but growing. Disproportionate risk aversion in the ambulance services has also been cited as a potential risk factor by O'Hara et al., (2015) and conveyance of patients to ED was considered the 'default safety net' because 'you don't lose your job from taking a patient to hospital' (Ibid, p. 49).

\section{Work intensity and implications for ambulance workers}

The introduction of the New Public Management (NPM) ideology into the UK NHS saw greater fiscal autonomy but more stringent regulations, control over managers and management by targets, impacting on work patterns (Bevan and Hamblin, 2009; Bevan and Hood, 2006) without conclusive proof about its usefulness and success (Smith, 1995). Issues concerning performance measurement, performance management and the usefulness of management by 'targets' approach have been widely debated in the public management literature (De Bruijn, 2002; Pollitt, 2003; Greiling, 2006). Wankhade (2011) has documented several unintended consequences of the performance regime in the UK ambulance services highlighting the potential costs of such schemes, the role of sub-cultural groups and the negative impact on workforce patterns. Failure to achieve targets and poor performance scores are seen as a personal failure of senior NHS leaders creating a climate of mistrust and conflict. Gascoigne et al., (2015, p. 461) provide a useful summary of some of these studies which highlight particular characteristics of work determining long and intense work hours and the occupational identity of the job holders. Green (2004) emphasises the various technological changes for modern work intensification. Studies on other emergency services including ambulance workers (Tangherlini, 2000; Palmer, 1983; Reynolds, 2009) and police (Van Maanen, 1978) further highlight such characteristics. 
Literature on occupational and professional 'identity' has explored how organisations and occupations influence and control people's work-related identities (Alvesson et al., 2008). Kuhn (2006, p. 1354) argues that the 'choices' actors make about time at work are shaped by the array of discursive possibilities available for identity construction rather than a result of any managerial manipulation. Actors employ accounts that align with their own self-narratives and the expectations of others in the workplace (Alvesson and Wilmont, 2002). Wankhade (2012) in his study classifying ambulance culture demonstrated how each of the occupational groups has their distinct assumptions and beliefs towards their roles and understanding about the service.

$\underline{\text { Implication of work intensity on sickness absence in the ambulance services }}$

Living a life on the edge (Hewitt and Luce, 2006) has its own perverse consequences. There is a growing discourse surrounding the various health related issues in the ambulance service with the underlying assumption that such work is inherently stressful (Hegg-Deloye et al., 2014; Coxon et al., 2016; Mishra et al., 2010; Sterud, et al., 2011, 2006, 2008; Bennett et al., 2004; Pajonk, et al., 2011; Nightingale and Mark, 1988, Clohessy and Ehlers, 1999; Alexander and Klein, 2001; Mahony, 2001). To this effect, Sterud et al., (2011, p.2) argue that ambulance personnel routinely attend non-emergency work, such as transporting and providing appropriate care to chronically and terminally ill patients, which imposes "different emotional demands and which might be experienced as more emotionally exhausting than more sensational events". Recently, Chatzitheochari and Arber (2009) highlighted the potential adverse health and safety consequences of sleep deprivation of British workers, citing evidence from the UK Time Use Survey. Ambulance professionals are regularly involved in rushing to 
the scene of emergency and taking rapid action often in very unstable and unfamiliar environment and very often in presence of public (Cydulka et al., 1989). Prevalence rates of burn-outs, divorce, suicide and drug-abuse have been reported to be particularly high in the emergency medical practitioners (Wagner et al., 2002; van der Ploeg and Kleber, 2003) highlighting specific stressors and the health related issues in the ambulance service.

There is also a contrary view suggesting that ambulance work is not inherently stressful or unique and many other emergency services such as the police or fire and rescue service may exhibit similar characteristics (Boudreaux and Mandry, 1996) and that ambulance personnel are usually resilient against stress and trauma (Orner et al., 2003). However others (Michie and Williams, 2003; Sterud et al., 2011) argue that high levels of distress amongst ambulance staff is mainly due to general organisational factors that are quite similar to the occupations with a heavy work demands which include long hours, workloads, pressure of work, lack of control over work and poor support from managers. In a recent study, Hegg-Deloye et al., (2014, p. 247) reviewing the current state of literature concluded that paramedics accumulate a set of risk factors, including acute and chronic stress, that can lead to the development of cardiovascular diseases and "post-traumatic disorders, sleep problems and obesity are prevalent among paramedics." Coxon et al., (2016) has found similar evidence of key stressors and their impact on well-being of staff working in the ambulance dispatch. Halpern et al., (2011, p.51) similarly argued that ambulance workers frequently deal with stressful or critical incidents that can result in significant 'emotional sequelae', such as depression, burnout and post-traumatic stress symptoms.

Sickness absence data in the UK NHS has been published by the Department of Health (DH) and Office for National statistics (ONS). This article relies on the statistical bulletins for 
sickness absence rates of NHS staff in England published by the Health \& Social Care Information Centre (HSCIC) using Electronic Staff Record (ESR). To our knowledge, such a synthesis is currently lacking in the literature.

The average sickness staff absence rates in NHS in England are 4.23 per cent annually over the four year period. The national annual sickness absence rate has fallen each year from 2009-10 to $2012-11$. It fell from 4.40 per cent in $2009-10$ to 4.16 per cent in $2010-11$ to 4.12 per cent in 2011-12. It then rose to 4.24 per cent in 2012-13. NHS workers in England took an estimated 9.5 working days off sick last year (HSCIC, 2013). The latest figures for 2012-13 compare to an estimated 9.3 working days in 2011-12 and 9.9 working days in 2009-10, the first year of reporting. The findings for the last four financial years relate to about 1.05 million full time equivalent workers in the English NHS (excluding GPs and practice staff) and are based on applying the measured sickness absence rate to an assumed full time working pattern of 225 days a year (ibid, p. 4).

\section{(Table1 here)}

Scrutiny of the sixteen quarterly reports reveals the following trends in relation to sickness absence of ambulance staff:

- Ambulance Staff were the staff group with the highest average sickness absence rate with an average of 6.24 per cent.

- Amongst types of organisation, Ambulance Trusts had the highest average sickness absence rate with an average of 5.76 per cent. Strategic Health Authorities had the lowest average with a rate of 2.76 per cent. These figures are significantly higher than the average national absence rates. 
- The figures for ambulance staff continue to show higher rate of absence. It is estimated that in 2012-13 average sick days per person equated to 14.7 days for qualified ambulance staff, compared to 13.9 in 2011-12 and 14.4 in 2009-10, significantly higher than national average.

- Ambulance sickness rates fell marginally only once in 2010-11 (from 5.73 per cent to 5.67 per cent) and continue to show rise during 2011-12 and 2012-13.

If one compares the sickness absence rates by Staff groups in the NHS, a similar picture emerges.

\section{(Table 2 here)}

Against the national average absence rate of 4.23 per cent over the four year period, ambulance staff shows an average absence rate of 6.32 per cent which is the highest amongst the entire NHS workforce included in the data set. The Boorman Review (2009) looking into the health and well-being of NHS staff recommended that the NHS Staff health and well-being needs should be seen as central to the NHS and recognised as a crucial issue at board level as much as at ward level. It further argued that staff health and well-being is not just the responsibility of occupational health departments or well-being advisers but it is the responsibility of every single member of staff.

These statistics need to be treated cautiously. The figures don't provide a split of the category of the ambulance workers showing sickness absence. Further, HSCIC does not currently publish causal data on specific reasons for sickness absence within the NHS in England and more specifically about the ambulance staff. Hence it is not possible to assign specific causes for such a high sickness absence rates for ambulance staff. The high levels of mental and health 
related issues identified with the ambulance workers earlier in this section (see Coxon et al., 2016; McCann et al., 2015; Hegg-Deloye et al., 2014; Halpern et al., 2011; Mishra et al., 2010) might allude, at least partially, to such high sickness rates but no such claims are made in this paper. The link between high sickness absence rates and stress levels in the ambulance workers is currently under-researched and by raising this issue, we hope to encourage further empirical research to understand the link better but such high absence rates are quite revealing to say the least. The next section details the research methodology adopted in this study.

\section{Data and methods}

The findings for this study are derived from a larger ethnographic study conducted in a large NHS ambulance trust in the North of England (Delta trust for confidentiality and anonymity). The main research question in the study was to explore the perceptions of the staff in dealing with the steady rise in ambulance demand amidst the changing clinical and educational scope of paramedic practice. Due to the exploratory nature of this study, the primary aim was to access the different experiences of a range of staff within the organisation in understanding their social settings (Van Maanen, 2011). Ethnographic studies thus have a considerable potential to play a larger and more mainstream role in organisation and management studies (Watson, 2011, p. 202).

Semi-structured interviews, non-participant observation and ambulance 'ride-ons' were used to gather data during 2007-2009. Board executives, managers and front-line staff (paramedics and control room) were interviewed. More than seventy interviews were conducted. The participants in the study included senior board executives, managers, and frontline staff- 
paramedics, the 999 call handlers and the dispatchers working in the control room. The selected sample helped to facilitate understanding of the social dynamics underlying the case.

About 100 hours of non-participant observation informed this study. Observation was carried at the corporate level; at the level of middle executives and managers, and frontline staffparamedics and control room, allowing for an analysis of the interdependence between these various organisational dimensions (Currie et al., 2008). This included attending open trust board meetings and few internal executive meetings were observed at the corporate level and four meetings with the managers. At the frontline, operations in the Control rooms were observed and time was also spent in the ambulance stations, travelling with ambulance crews and in the canteen where managers, junior executives, and frontline staff took breaks. Observation was complemented with informal conversations in the 'corridors' with staff. Conversations typically related to specific issues observed in the interviews, nature of work and discussion of the context that framed the decision on the matters while allowing a better understanding of the dynamics of the nature of work in the case study organisation (Yin, 2009).

Interview and observed data were transcribed and the responses to the key themes were analysed repeatedly to further explore the response of the participants to the main research question (Rhodes and Brown, 2005; Boje, 1991; Sayer, 2000; Charmaz, 2003). This methodology provided insights into how individual 'behaviours' of various institutional actors were shaped, how these decisions and actions that individuals made during their working (\& non-working hours) were socially constructed and constituted in performance of their specific roles within the organisation. Ethical approval for the larger study was granted by an NHS research ethics committee. 


\section{Findings and results}

Interviews with some frontline staff revealed a different perspective about the core values and mission of the organisation:

"A lot of our staff don't think going to patients with minor things and leaving them at home is a worthwhile thing to have done. What our staff, in the way that they were recruited, they're recruited to be, if you like, the adrenalin junkies, they get there, they save someone's life, they drive somewhere else, deliver the patient to a team that's all... it's like the telly isn't it? That's why people join"

Senior Board Executive I

\footnotetext{
"You come for an interview for the ambulance service - you've seen Casualty on TV and Holby City and that's the perception that is continually in the mind of the candidates for the role...And the disappointment comes when they achieve that role and it isn't everything they thought it would be."
}

\section{Senior Paramedic I}

It is important to emphasise that since the training for paramedics is now at the level of a university graduation, staff who have worked for long in the ambulance services can be wary of the new university-trained recruits, and might feel the new cadre has advanced too quickly without really understanding 'the realities of the road'. Indeed, the paramedic science degree has given them an exit route to work outside of the ambulance services, something which didn't really exist for the 'old school' ambulance workers who have 'come up' through the ranks. Evans et al., (2014) in their systematic review of the literature examined the evidence of paramedics trained with extra skills and the impact on patient care. The review identified many 
viable extra skills for paramedics but concluded that the evidence is "not strong enough to guide policy." (p. 602). It is also the case that ambulance services are facing a shortage of paramedics as many new university educated staff are leaving the service feeling disillusioned and unsupported something which was highlighted in a recent inspection report (Care Quality Commission, 2015, p.41:

"Several frontline staff reported morale to be low due to 'workload pressures'. These included a high number of resignations, mental exhaustion, lack of career progression, lack of feedback from managers, long working hours, pay scale in comparison to other services and lack of recognition for hard work."

Ambulance work patterns are governed by the Working Time Regulations (WTR) which specifies a maximum 48 hour weekly limit. Most frontline staffs, especially paramedics, routinely work in excess of these hours in form of extended shifts or overtimes. The public perception about the ambulance staff also plays some part in heightening such tensions. MORI polls score the ambulance staff very high in the user satisfaction. But it has its own unintended consequences and quite a few respondents complained about the 'burden' of such high expectations from the public:

"My colleagues don't view performance because one of the major features in the ambulance service is one job at a time. They don't care at the end of the day what those figures say. It's because the minute they come here for a 12 hour shift, whether it be a day shift or a night shift, it's a job after job."

Senior Paramedic II

One senior executive seemed to agree with this assessment:

"I suspect that given the nature of the service, the public are just grateful that the ambulance turned up and got them to hospital. I've been out with crews and people feel you can do no 
wrong. There are a lot of assumptions from people who might never have been in an ambulance before."

\section{Senior Board Executive II}

Recent evidence support these findings. A rapid review by Turner et al., 2015, examined the evidence on the effectiveness of different models of delivering urgent care, concluded a "lack of evidence about the implications of developing the paramedic workforce at scale to achieve national objectives (p.66)." O'Hara et al., (2015) in their study of systemic influences on paramedic decision-making reported seven overarching system influences while identifying them as potential risk factors. Increased demand for ambulance service care was reported to have impacted on the scope of clinical decision making by paramedics as the "profile of calls has shifted from primarily emergency care decisions to now dealing with a wider range of primary care and psychosocial decisions" (Ibid, p. 48). Performance regime and priorities, especially the eight minute response time target was also regarded as a source of pressure for staff and resources, particularly when calls were not life-threatening and also confirmed in another study (Wankhade, 2011). Fisher et al., (2015, p. iv) in their scoping review about patient safety in ambulance services, identified many reports of cases for which operational pressures, including targets, were perceived to be more important than patient safety for ambulance services.

The study also recorded perceptions about the challenge for the ambulance services as being seen merely as a transport service to a professional healthcare provider (Heath and Wankhade, 2014; McCann et al., 2013; Wankhade, 2011):

"Our predominant staff are not professionalised in the way that you would expect the clinical profession to be and that's because profession has happened to them - they haven't joined a 
profession. They didn't have to acquire anything to achieve to become that professional in the first place."

\section{Senior Clinician}

With the current recruitment practises, these perceptions are changing. The new specialist paramedic roles as outlined earlier in the article have helped ambulance services reduce conveyance of patients to ED and saving costs. But absence of effective alternative care pathways has also been identified in several recent studies (Fisher et al., 2015; Turner et al., 2015; Evans et al., 2014) with reported lack of clear evidence base and considerable variations in the deployment of paramedics with extended skills in managing patients with urgent health problems in the community settings and/or dispatched to unsuitable patients. Disproportionate risk aversion in the ambulance services has also been cited by O'Hara et al., (2015) and conveyance of patients to ED was considered the 'default safety net' because 'you don't lose your job from taking a patient to hospital' (Ibid, p. 49).

Many staff expressed frustration about lack of meaningful opportunities for staff training and development. One common comment made during informal discussions was how staff training was seen secondary to meeting the operational exigencies and performance pressures. Recent evidence confirms our findings (Turner et al., 2015; O'Hara et al., 2015; Fisher et al., 2015; Mackway-Jones and Wankhade, 2015). 
Senior executives when questioned about high sickness absence rates for ambulance staff did not deny the emotional challenges of the job but were quick to point out the support available to staff for dealing with sickness issues. One senior manager however raised few concerns:

"Sickness can rise because people are not being seen about absence and the sickness policy isn't adhered to which can have some quite serious consequences... They may then be back in work for a couple of days, but be off sick again with the same problem which could have been dealt earlier."

\section{Senior Operational Manager I}

Some of these comments can be explained in the way ambulance trusts deploy their paramedic staff. Ambulance crews usually work in pairs and rotate together in their twelve hour shifts or sometimes work alone in remote ambulance stations or solo in rapid response vehicles (RRVs). High level of sickness-absence rates, lack of capacity and low staff morale was also highlighted recently (McCann et al., 2015). There is little opportunity for training or managerial support during shifts. As a consequence, clinical supervision gets sacrificed when everybody has to go out. And it ceases to be an organisational priority. This often acts as barrier to communication and support. Concerns over the utilisation of reporting and feedback mechanisms, dearth of routine and constructive feedback on clinical decisions made by paramedics were also highlighted recently (O’Hara et al., 2015).

There is a growing debate about the lack of clinical evidence base behind response time targets which is putting the crews and patients at risk (NHS Confederation, 2014) though a more broad based targets regime is now operational (Cooke, 2011). Evidence suggests 
that the use of lights and sirens improves the ambulance response time by an average of 1 minute, 46 seconds which is clinically relevant in only a few cases (Brown et al., 2000).

Over-prioritisation of 8 minute red calls (response to a life-threatening emergency) came up as another reason for crew dissatisfaction. American paramedics in Palmer's ethnography (1983:167) refer to such calls as "211s versus Pukes" in differentiating between 'good runs, saves, celebrated cases (211s) versus non-essential calls and nuisance calls (Pukes). Crews talked about their frustration in rushing and speeding to such calls which turned out to be less serious incidents not deserving such rushed response:

"A lot of falls now are red responses. And because it is a very risk-averse tool with lot of default settings to go red, it doesn't necessarily instil faith amongst the crews that are then responding to a call. You've got non clinical staff using a risk averse, non-clinical triage tool at the call stage to inform a clinical response."

\section{Senior Operational Manager II}

"My last job was chest pain for a 65 year old. So I go in with two very heavy bags and they are very heavy, especially after 12 hours, up to the second floor with no lift and this guy had stomach pain. I mean not chest pain but that was a Red call and it was because of the information given to the people and the way they ask their questions. So I feel in many respects we make work for ourselves. Red calls by their nature sometimes put words into people's mouths i.e. "Do they have chest pain?" "Oh yes." "Are they breathless?" and they're going to say, yes because the public then know that the ambulance might get there a little bit quicker". 
Two such incidents happened during the ambulance 'ride-ons' by the author. On reaching to a patient (999 call) on a Saturday night at around $6.30 \mathrm{pm}$, the caller-an old person aged about 70 years was waiting outside his house with a bag for the ambulance to arrive. The crews knew him since he had a history of cardiac problems in the past but on this occasion he simply did not feel safe to be alone on a weekend and insisted to be taken to the hospital. In the second instance, a twenty year old caller had complained about breathlessness and dialled 999 for an urgent ambulance response after running out of his inhaler. It is true that any such system can be open to abuse. It was evident that the extent and increased demand was a notable source of strain on staff and organisation.

Historically and in the new commissioning framework since April 2013, performance (and payment) of individual ambulance trusts is still guided by the achievement of the 8 minute response time target. Limitations of such an approach which focus on treating the clock, rather than the patient have informed current debates (Wankhade and Mackway-Jones, 2015; McCann et al., 2013; Siriwardena et al., 2010). Increased demand coupled with staff shortage and incorrect dispatch of staff (O'Hara et al., 2015) can result into a culture of fear amongst frontline staff (CQC, 2015, p. 41). Similar issues about the role of target culture and its impact on behaviour of frontline staff was also recorded in other studies (Wankhade et al., 2015; McCann et al., 2013; Wankhade and Brinkman, 2014; Wankhade, 2012). Recent evidence has also pointed a gap between the

\section{Discussion and conclusion}


A multiple-level analysis has provided consistent evidence around the issues explored in this study. The study findings provide insight into the different perceptions of staff towards the changing scope and practise of the ambulance staff and the growing pressures of operational demand. This study also makes contribution through providing insights into the intraorganisational dynamics in an unusual organisational setting. The study highlights the challenges faced by ambulance services in coping with the increased demand and emerging paramedic specialist roles. Findings from this study also point to lack of uniformity about the new roles and their appropriate dispatch to the right patients. Attention has also been drawn to the inconclusive evidence about the safety of patents who have not been conveyed to the EDs and the complexity of the decision-making process to minimise potential risks to patients.

Recent evidence support our study findings (Bigham et al., 2012; Hill et al., 2014; Tohira et al., 2013) and also the fact that these specialist paramedic roles with higher level of education and training represents a 'minority' of paramedic staff (O'Hara et al. 2015, p. 52). Role of system factors such as increased demand, performance regimes and the role of 8 minute response time target, lack of training and education opportunities and variations in alternate care pathways having significant implications for patient safety has been recorded in our study which are significant findings and are supported in a recent scoping study on patient safety in ambulance services (see Fisher et al., 2015) and rapid review on effectiveness of different models of delivering urgent care (Turner et al., 2015).

These findings also have significant implications for employment practices. It raises questions about whether it is possible and sustainable to motivate staff and/or retain and recruit new members while moderating these negative influences. Further empirical work can shed more light on these aspects. Development of strategies to prevent significant levels of sickness and 
occupational health issues, to combat prevalence of general and specific ambulance specific stress factors follows our analysis and is supported in the literature. High levels of emotional exhaustion, often accompanied by lack of managerial and peer support with musculoskeletal pain (physical demands) which was identified by many respondents as ambulance specific drivers of stress is also supported in the literature (Hegg-Deloye et al., 2014; Sterud et al., 2011, p. 8). Benett et al., (2011) provide a good analysis of personality traits of emergency physicians and paramedics in influencing their behaviour and decision-making. With numerous media reports about the increase in the number of cases of assaults on ambulance crews, there are real concerns around safety of the crews, especially for paramedics who work on the RRVs. Management literature linking health impact to high sickness absence levels in ambulance workers is quite sparse and needs further investigation, something which is highlighted in our study. Understanding the personality characteristics of ambulance workers which may predispose them to develop these disorders has further been suggested (Pajonk et al., 2011; Clohessy and Ehlers, 1999). Surprisingly the literature linking sickness issues to high sickness absence rates in ambulance workers is quite sparse and needs further investigation as highlighted by our study. A recent efficiency review (Carter, 2015) expressed concerns of the high level of sickness absence rates in the NHS ranging from $3.1 \%$ to $5 \%$ and estimated that a $1 \%$ improvement in staff productivity will save the NHS $£ 280$ million a year, which equates to hospitals using new working methods that would save every member of staff 5 minutes on an 8 hour shift.

Ambulance services are witnessing a period of turbulence. Amidst the progress on the professionalisation of the paramedic agenda, individual trusts are facing challenges in form of staff attitudes towards meeting performance targets, coupled with rising demand, fear of loss of contracts and private competition (Mackway-Jones and Wankhade, 2015; McCan et al., 
2015). Ambulance services, it has been recently argued, remain 'locked in an 18th Century mind-set' that reinforces a traditional emergency care and transport focused mode of operation, which is insufficiently attuned to the changing and more heterogeneous actualities of demand of the modern world (Newton and Harris, 2015).

It is worthwhile noting some of the limitations of this paper. The study provides a thick description of the case settings in an English NHS ambulance trust and the readers are better able to assess how well these findings may apply in other contexts (Lincon and Guba, 1985). The evidence cited is specific to English ambulance services was gathered during 2007-2009 and further empirical research will be valuable to test the relevance of these findings in a variety of settings. Recent evidence as cited in the article supports our study findings. The paramedic education in the UK has moved towards a university level qualification since the study period but the evidence gathered in the study is relevant to the issues raised in the recent studies examining leadership and management challenges, lack of a conclusive evidence base about patient safety and systemic influences on paramedic decision making (McCann et al., 2013; Fisher et al., 2015; O’Hara et al., 2015; Turner et al., 2015; Newton and Harris, 2015; Evans et al., 2014). Globally, ambulance services are facing similar challenges surrounding rising activity, a growing older population, societal and cultural issues and a drive for professionalisation of practice leading to similar cultural challenges for evolving into a professional healthcare providers (Vincent-Lambert, 2015; Jormakka and Saikko, 2015; Hou, et al., 2013). Findings from this study thus have international implications and can be relevant in other settings equally.

To conclude, the relationship between high sickness levels and ambulance work patterns highlighted in our study need greater management and policy attention in different settings than 
it currently does. Similarly, the implications of work intensity on gender equality within ambulance settings remain unexplored (Burke and Fiksenhaum, 2009; Kelly et al., 2010). Effectiveness of the paramedic work patterns and implications for organisational productivity needs further investigation. Empirical studies in different settings exploring the impact of work intensity on social environment (Pfeffer, 2010) including the impact on human sustainability (Schor, 2011) can further provide a fruitful research agenda.

\section{References}

Alexander, D.A. and Klein, S. (2001). Ambulance personnel and critical incidents: impact of accident and emergency work on mental health and emotional well-being. British Journal of Psychiatry 178, pp. 76-81.

Ambulance Service Association (2000). The Future of Ambulance Services in the United Kingdom: A strategic review of options for the future of ambulance service, Medical care Research Unit, The University of Sheffield: Sheffield.

Alvesson, M., Ashcroft, K.L. and Thomas, R. (2008). Identity Matters: Reflections on the Construction of Identity Scholarship in Organization studies. Organization, 15, 1, pp. 5-28.

Alvesson, M. and Willmott, H. (2002). Identity Regulation as Organizational Control: Producing the Appropriate Individual. Journal of Management Studies, 39, 5, pp. 619-644.

Andrews, R. and Wankhade, P. (2015). Regional Variations in Emergency Service Performance: Does Social Capital Matter?, Regional Studies, 49, 12, pp. 2037-2052.

Anteby, M. (2013). Relaxing the Taboo on Telling Our Own Stories: Upholding Professional Distance and Personal Involvement. Organization Science, 24, 4, pp. 1277-90.

Association of Ambulance Chief Executives (2011). Taking Healthcare to the Patient 2: A review of 6 years' progress and recommendations for the future. AACE: London. 
Bennett, P., Williams, Y., Page, N. et al. (2004). Levels of mental health problems among UK emergency ambulance workers. Emergency Medicine Journal, 21, pp. 235-236.

Bevan, G. and Hood, C. (2006). What's Measured is What Matters: Targets and Gaming in the English Public Health Care System. Public Administration, 84, 3, pp. 17-38.

Bevan, G. and Hamblin, R. (2009). Hitting and missing targets by ambulance services for emergency calls: effects of different systems of performance measurement within the UK. Journal of the Royal Statistical Society, 172, 1, pp. 161-90.

Bigham BL, Buick JE, Brooks SC, et al. (2012). Patient safety in emergency medical services: a systematic review of the literature. Prehospital Emergency Care, 16, pp. 20-35.

Boje, D. (1991). Constituting change in the storytelling organization. Journal of Organizational Change Management 4, 3, pp. 7-17.

Boorman, S. (2009). NHS Health and Well-being Review: Interim and Final Report, Department of Health: London.

Boudreaux, E. and Mandry, C. (1996). The effects of stressors on emergency medical technicians (Part II): A critical review of the literature, and a call for further research. Prehospital Disaster Medicine, 11, pp. 302-307.

Brown, L., Whitney, C., Hunt, R., Addario, M., and Hogue, T. (2000). Do Warning Lights and Sirens Reduce Ambulance Response Times. Prehospital Emergency Care, 4, 1, pp. 70-74.

Burke, R.J. (2009). Working to Live or Living to Work: Should Individuals and Organizations Care? Journal of Business Ethics, 84, pp. 167-172.

Burke, R. J. and Fiksenbaum, L. (2009). Are managerial women in "extreme jobs" disadvantaged? Gender in Management: An International Journal, 24, 1, pp. 5-13. 
Care Quality Commission (2015). London Ambulance Service NHS Trust: Quality Report, CQC: London.

Carter, L. (2016). Operational productivity and performance in English NHS acute hospitals: Unwarranted variations: An independent report for the Department of Health by Lord Carter of Coles. Her Majesty's Government: London.

Charmaz, K. (2003). Qualitative Interviewing and Grounded Theory Analysis in J.A. Holstein and J.F. Gubrium (eds.) Inside interviewing: new lenses, new concerns, Sage.: London.

Chatzitheochari, S. and Arber, S. (2009). Lack of sleep, work and long hours culture: evidence from the UK Time Use Survey. Work, Employment \& Society, 23, 1, pp. 30-48.

Clohessy, S. and Ehlers, A. (1999) 'PTSD symptoms, response to intrusive memories and coping in ambulance service workers', British Journal of Clinical Psychology 38:251-265.

Cooke, M. (2011). An Introduction to the New Ambulance Clinical Quality Indicators. Ambulance Today, 8, 1, pp. 35-39.

College of Paramedics (2015). Paramedic Curriculum Guidance. Third edition. The College of Paramedics: Derby.

College of Paramedics (2008). Paramedic Curriculum Guidance and Competence

Framework. 2nd edition. The College of Paramedics: Derby.

Coxon, A., Cropley, M., Schofield, P., et al., (2016). 'You're never making just one decision': exploring the lived experience of ambulance Emergency Operations Centre personnel. Emergency Medicine Journal. Published online first. March 2016.

Currie, G., Waring, J., and Finn, R. (2008). The Limits of Knowledge Management for UK Public Services Modernization: The Case of Patient Safety and Service Quality. Public Administration 86, 2, pp. 363-385. 
Cydulka, R.K., Lyons, J., Moy, A., Shay, K. et al. (1989). A follow-up report of occupational stress in urban EMT-paramedics. Annals of Emergency Medicine, 18, pp. 1151-1156.

Darzi A. (2008). High quality care for all: NHS next stage review final report. Department of Health: London.

De Bruijn, H. (2002). Managing Performance in the Public Sector, Routledge: London.

Department of Health, (2005). Taking Healthcare to the Patient; Transforming NHS Ambulance Services. Department of Health: London.

Evans, R., McGovern, R., Birch, J. and Newbury-Birch, D. (2014). Which extended paramedic skills are making an impact in emergency care and can be related to the UK paramedic system? A systematic review of the literature. Emergency Medicine Journal, 31, pp. 594603.

Fisher JD, Freeman K, Clarke A, et al. (2015). Patient safety in ambulance services: a scoping review. Health Service and Delivery Research. 3, 21. Available at http://dx.doi.org/10.3310/hsdr03210.

Gascoigne, C., Parry, E. and Buchanan, D. (2015). Extreme work, gendered work? How extreme jobs and the discourse of 'personal choice' perpetuate gender inequality. Organization, 22,4, pp.457-475.

Green, F. (2008). Work effort and worker well-being in the age of affluence', in R.J. Burke and C.L. Cooper (eds.) The long work hours culture: causes, consequences and choices, pp. 115-126, Emerald: Bingley.

Green, F. (2004). Why has work effort become more intense? Industrial Relations, 43, 4, pp. 709-741.

Greiling, D. (2006). Performance measurement: a remedy for increasing the efficiency of public services?, International Journal of Productivity and Performance Management, 55, 6, pp. 448-465. 
Halpen, J., Maunder, R.G., Schwartz, B. and Gurevich, M. (2011). Identifying risk of emotional sequelae after critical incidents. Emergency Medicine Journal, 28, pp. 51-56.

Halter, M., Vernon, S., Snooks, H., Porter, A., Close, J., Moore, F., and Porsz, S., (2011). Complexity of the decision-making process of ambulance staff for assessment and referral of older people who have fallen: a qualitative study. Emergency Medicine Journal, 28, pp. 44-45.

Health \& Social Care Information Centre (2014). NHS Sickness Absence Rates January 2014 to March 2014 and Annual Summary 2009-10 to 2013-14. Health \& Social Care Information Centre: London. (Accessed on 4 January 2016).

Health \& Social Care Information Centre (2014). NHS Sickness Absence Rates, Annual Summary Tables, 2009-10 to 2013-14 (Tables 2 \& 3). London: Health \& Social Care Information Centre: London. (Accessed on 4 January 2016).

Heath, G. and Radcliffe, J. (2010). Exploring the utility of current performance measures for changing roles and practices of ambulance paramedics. Public Money \& Management, 30, 3, pp. 151-158.

Heath, G and Radcliffe, J. (2007). Performance Measurement and the English Ambulance service. Public Money and Management, 27,3, pp. 223-27.

Heath, G. and Wankhade, P. (2014). A Balanced Judgement?: Performance Indicators, Quality and the English Ambulance Service; some issues, developments and a research agenda. The Journal of Finance and Management in Public Services, 13, 1, pp. 1-17.

Hegg-Deloye, S., Brassard, P., Jauvin, N., et al., (2014). Current state of knowledge of posttraumatic stress, sleeping problems, obesity and cardiovascular disease in paramedics. Emergency Medicine Journal, 31, pp. 242-247.

Hewitt, S.A. and Luce C.B. (2006). Extreme Jobs: The Dangerous Allure of the 70-Hour Workweek. Harvard Business Review, 84, 12, pp. 49-59. 
Hill H, McMeekin P and Price C. (2014). A systematic review of the activity and impact of emergency care practitioners in the NHS. Emergency Medicine Journal, 10, 853-860.

Hou, X., Rego, J. and Service, M. (2013). Paramedic education opportunities and challenges in Australia. Emergency Medicine Australasia, 25, 2, pp. 114-119.

Jormakka, J. and Saikko, S. (2015). International Perspectives: Finnish Ambulance Services in 2020 in P. Wankhade and K. Mackway-Jones (eds.) Ambulance Services: Leadership and Management Perspectives, Springer: New York.

Kelly, E. L., Ammons, S.K., Chermac, K. and Moen, P. (2010). Gendered Challenge, gendered Response: Confronting the Ideal Worker Norm in a White-Collar Organization. Gender \& Society, 24, 3, pp. 281-303.

Klein, K.J, Ziegert, J.C., Knight, A.P., and Xiao, Y., (2006). Dynamic Delegation: Shared, Hierarchical, and Deindividualized Leadership in Extreme Action Teams. Administrative Science Quarterly, 51, 4, pp. 590-621.

Kuhn, T. (2006). A 'Demented Work Ethic' and a 'Lifestyle Firm': Discourse, Identity, and Workplace Time Commitments. Organization Studies, 27, 9, pp. 1339-58.

Lincoln, Y.S. and Guba, E.G. (1985), Naturalist Inquiry, Sage: London.

Lovegrove, M. and Davis, J. (2013). Paramedic Evidence Based Education Project (PEEP): End of Study Report. UK: Allied Health Solutions.

Mackway-Jones, K. and Wankhade, P. (2015). Future Perspectives for the UK Ambulance Services: Evolution rather than Revolution. in P. Wankhade and K. Mackway-Jones (eds.) Ambulance Services: Leadership and Management Perspectives, Springer: New York. pp.157-162.

Mahony, K. L. (2001). Management and the creation of occupational stressors in an Australian and a UK ambulance service. Australian Health Review, 24, pp. 135-145. 
Mannon, J. M. (1992). Emergency Encounters: EMTs and Their Work. Jones and Bartlett: Boston, MA.

McCann, J., Granter, E., Hassard, J. and Hyde, P. (2015). Where next for the Paramedic Profession? An Ethnography of Work Culture and Occupational Identity. Emergency Medicine Journal, 32, pp. e6-e7.

McCann, J., Granter, E., Hyde, P. and Hassard, J. (2013). Still Blue-Collar after all these Years? An Ethnography of the Professionalization of Emergency Ambulance Work. Journal of Management Studies, 50, 5, pp. 750-776.

McCann, L., Hassard, J. and Morris, J.L. (2008). Normalized Intensity: The New Labour Process of Middle Management. Journal of Management Studies, 45, 2, pp. 343-71.

Metz, D. L. (1981). Running Hot: Structure and Stress in Ambulance Work. ABT Books: Cambridge, MA.

Michie, S. and Williams, S. (2003). Reducing work related psychological ill health and sickness absence: a systematic literature review. Occupational Environment Medicine Journal, 60, pp. 3-9.

Mishra, S., Goebart, D., Char, E., Dukes, P. and Ahmed, I. (2010). Trauma exposure and symptoms of post-traumatic stress disorder in emergency medical services personnel in Hawaii. Emergency Medicine Journal, 27, pp. 708-711.

Newton, A. and Harris, G. (2015). Leadership and System Thinking in the Modern Ambulance Service in P. Wankhade and K. Mackway-Jones (eds.) Ambulance Services: Leadership and Management Perspectives, Springer: New York. pp. 81-94.

NHS Five Year Forward View (2014). NHS England: Leeds.

NHS England, (2013). Transforming Urgent and Emergency Care in England: Urgent and Emergency Care Review, Phase 1 Report, NHS England: Leeds. 
National Audit Office (2010). Major trauma care in England, HC 213, Session 2009-2010. NAO: London.

NHS Confederation, (2015). Rip off the sticking plaster now: Enabling the local implementation of sustainable urgent and emergency care models in 2015/16, NHS Confederation: London.

NHS Confederation, (2014). Ripping off the sticking plaster: Whole-systems solutions for urgent and emergency care, NHS Confederation: London.

NHS Confederation (2011). Critical care paramedics: delivering enhanced pre-hospital trauma and resuscitation care: a cost-effective approach. NHS Confederation: London.

NHS Modernisation Agency (2004). Driving Change: Good Practice Guidelines for PCTs on Commissioning Arrangements for Emergency Ambulance Services \& Non-Emergency Patient Services. NHS Modernisation Agency: London.

Nightingale, S.D. and Grant, M. (1988). Risk preference and decision making in critical care stations. Chest, 93, pp. 684-7.

O’Hara, R., Johnson, M., Siriwardena, A.N. et al., (2015). A qualitative study of systemic influences on paramedic decision making: care transitions and patient safety. Journal of Health Services Research \& Policy, 20(Suppl. 1), pp. 45-53.

Orner, R.J. (2003). 'A new evidence base for making early intervention in emergency services complimentary to officers' preferred adjustment and coping strategies', in R.J. Orner and U. Sohnyder (ed.) Reconstructing early intervention after trauma. Oxford University Press:Oxford.

Pajonk, F-G., Andresen, B., Schneider-Axmann, T., Teichmann, a. et al. (2011). Personality traits of emergency physicians and paramedics. Emergency Medicine Journal, 28, pp. 141146. 
Palmer, C. E. (1983). "Trauma Junkies" and Street Work: Occupational Behavior of Paramedics and Emergency Medical Technicians. Journal of Contemporary Ethnography, 12, 2, pp. 162-83.

Pfeffer, J. (2010). Building Sustainable Organizations: The Human Factor. Academy of Management Perspectives, 24, 1, pp. 34-45.

Pollitt, C. (2003). The Essential Public Manager, Open University Press: Maidenhead.

Porter, A., Snooks, H., Youren, A., Gaze, S. et al. (2007). 'Should I stay or should I go?' Deciding whether to go to hospital after a 999 call. Journal of Health Services Research \& Policy, 12, 1, pp. 32-38.

Price, L. (2006). Treating the Clock and Not the Patient: Ambulance Response Times and Risk. Quality and Safety in Health Care, 15, pp. 27-30.

Reynolds, T. (2009). Blood, Sweat and Tea: Real-Life Adventures in an Inner-City Ambulance, The Friday Project: London.

Rhodes, C. and Brown, A. (2005). Narrative, Organisations and Research. International Journal of Management Reviews, 7, 3, pp.167-188.

Sayer, A. (2011). Realism and Social Science. Sage: London.

Schor, J. (2011). True Wealth, Penguin: London.

Siriwardena, A.N., Donohoe, R., Stephenson, J. et al., (2010). Supporting research and development in ambulance services: research for better health care in prehospital settings. Emergency Medicine Journal, 27, pp. 324-326.

Skolnick, J.H. (2008). Enduring issues of police cultures and demographics. Policing \& Society 18,1, pp. $35-45$. 
Smith, P.C. (1995). On the Unintended Consequences of Publishing Performance Data in the Public Sector. International Journal of Public Administration, 18, 2/3, pp. 277-310.

Snooks, H., Evans, A., Wells, B., Peconi, J., Thomas, M., Woollard, M., Guly, H., Jenkinson, E., Turner, J. and Hartley-Sharpe, C. (2009). What are the highest priorities for research in emergency prehospital care? Emergency Medicine Journal, 26, pp. 549-550.

Sterud, T., Hem, E., Lau, B. and Ekeberg, O. (2011). A comparison of general and ambulance specific stressors: predictors of job satisfaction and health problems in a nationwide oneyear follow-up study of Norwegian ambulance personnel. Journal of Occupational Medicine and Toxicology 6, 10. pp.1-9.

Sterud, T., Hem, E., Ekeberg, O. and Lau, B. (2008). Occupational stressors and its organizational and individual correlates: A nationwide study of Norwegian ambulance personnel. BMC Emergency Medicine 8, 16, pp. 1-11.

Sterud, T., Ekeberg, O. and Hem, E. (2006). Health status in the ambulance services: a systematic review. BMC Health Services Research, 6,82, pp. 1-10 .

Tangherlini, T. L. (2000). Heroes and Lies: Storytelling Tactics among Paramedics. Folklore, 111, pp. 43-66.

Tohira H, Williams TA, Jacobs I, et al. (2013). The impact of new prehospital practitioners on ambulance transportation to the emergency department: a systematic review and metaanalysis. Emergency Medicine Journal, 26, pp. 88-94.

Turner, J., Coster, J., Chambers, D., et al., (2015). What evidence is there on the effectiveness of different models of delivering urgent care? A rapid review. Health Services and Delivery Research, 3, 43, pp.1-133.

Van Maanen, J. (2011). Ethnography as Work: Some Rules of Engagement. Journal of Management Studies, 48,1, pp. 218-234. 
Van Maanen, J. (1978). 'The Asshole' in P.K. Manning and J. Van Maanen (eds.) Policing: A View from the Street, Random House: New York. pp. 221-238.

van der Ploeg, E. and Kleber, R.J. (2003). Acute and chronic job stressors among ambulance personnel: predictors of health symptoms. Occupational Environment Medicine, 60, pp. 4046.

Vincent-Lambert, C. (2015). International Perspectives: South African Ambulance Services in 2020 in P. Wankhade and K. Mackway-Jones (eds.) Ambulance Services: Leadership and Management Perspectives, Springer: New York. pp. 175-184.

Wagner, D., Heinrichs, M. and Ehlert, U. (2002). Prevalence of symptoms of posttraumatic stress disorder in German professional firefighters. American Journal of Psychiatry 155, pp. 1727-32.

Wankhade, P. and Mackway-Jones, K. (eds.) (2015). Ambulance Services: Leadership and Management Perspectives, Springer: New York.

Wankhade, P. and Brinkman, J. (2014). The negative consequences of culture change management: evidence from a UK NHS ambulance service. International Journal of Public Sector Management, 27, 1, pp. 2-25.

Wankhade, P. (2012). Different Cultures of Management and their Relationships with Organisational Performance: Evidence from the UK Ambulance Service. Public Money \& Management, 32, 2, pp. 381-388.

Wankhade, P. (2011). Performance Measurement and the UK Emergency Ambulance Service: Unintended Consequences of the Ambulance Response Time Targets. International Journal of Public Sector Management, 24, 5, pp. 382-402.

Wankhade, P. (2011a). Emergency Services in Austerity: Challenges, Opportunities and Future Perspectives for the Ambulance Service in the UK. Ambulance Today, 8,5, pp. 13-15. 
Wankhade, P. and Brinkman, J. (2011). 'Dilemmas of ambulance professionals in attending to an emergency within eight minutes: The ethics of target setting', in D.T.H, Weir and N. Sultan (ed.) From Critique to Action: The Practical Ethics of the Organisational World, , Cambridge Scholars: Newcastle-Upon-Tyre. pp. 106-129.

Watson, T. (2011). Ethnography, Reality and Truth: The Vital Need for Studies of 'How Things Work' in Organizations and Management. Journal of Management Studies, 48, 1, pp. 202-217.

Woollard, M. (2009). Professionalism in UK paramedic practice. Australian Journal of Paramedicine, 7, 4, Article 9.

Yin, R. K. (2009). Case Study Research: Design and Methods, Sage: London. 
Table 1: Annual Sickness Absence Rates by Organisation Type in the NHS

\begin{tabular}{|l|l|l|l|l|l|}
\hline & $2009-10$ & $2010-11$ & $2011-12$ & $2012-13$ & $2013-14$ \\
\hline England & $\mathbf{4 . 4 0 \%}$ & $\mathbf{4 . 1 6 \%}$ & $\mathbf{4 . 1 2 \%}$ & $\mathbf{4 . 2 4 \%}$ & $\mathbf{4 . 0 6 \%}$ \\
\hline Acute trusts & $4.18 \%$ & $3.91 \%$ & $3.89 \%$ & $4.01 \%$ & $3.84 \%$ \\
\hline Ambulance trusts & $5.73 \%$ & $5.67 \%$ & $5.76 \%$ & $6.05 \%$ & $5.82 \%$ \\
\hline Clinical Commissioning Groups & - & - & - & $2.07 \%$ & $2.20 \%$ \\
\hline Commissioning Support Groups & - & - & - & - & $2.69 \%$ \\
\hline Community Provider Trusts & $4.07 \%$ & $4.64 \%$ & $4.60 \%$ & $4.65 \%$ & $4.47 \%$ \\
\hline Mental Health & $5.23 \%$ & $4.95 \%$ & $4.89 \%$ & $4.94 \%$ & $4.74 \%$ \\
\hline PCT & $4.42 \%$ & $4.20 \%$ & $3.93 \%$ & $3.09 \%$ & $3.26 \%$ \\
\hline Special Health Authority & $3.93 \%$ & $3.69 \%$ & $3.47 \%$ & $3.56 \%$ & $3.30 \%$ \\
\hline SHA & $2.22 \%$ & $2.31 \%$ & $2.13 \%$ & $2.55 \%$ & - \\
\hline
\end{tabular}

Source: Adapted from NHS Sickness Absence Rates, Annual Summary Tables, 2009-10 to 2013-14, Health and Social Care Information Centre. 2014 (Table 2)

Table 2: Annual Sickness Absence Rate by Staff Groups in the NHS

\begin{tabular}{|l|l|l|l|l|l|}
\hline & $2009-10$ & $2010-11$ & $2011-12$ & $2012-13$ & $2013-14$ \\
\hline Total & $\mathbf{4 . 4 0 \%}$ & $\mathbf{4 . 1 6 \%}$ & $\mathbf{4 . 1 2 \%}$ & $\mathbf{4 . 2 4 \%}$ & $\mathbf{4 . 0 6 \%}$ \\
\hline $\begin{array}{l}\text { Professionally qualified clinical } \\
\text { staff }\end{array}$ & $3.85 \%$ & $3.65 \%$ & $3.61 \%$ & $4.24 \%$ & $4.06 \%$ \\
\hline All HCSC doctors & $1.21 \%$ & $1.16 \%$ & $1.19 \%$ & $1.25 \%$ & $1.22 \%$ \\
\hline $\begin{array}{l}\text { Total HCHS non-medical staff } \\
\text { Qualified nursing, midwifery \& } \\
\text { health wellbeing staff }\end{array}$ & $4.72 \%$ & $4.46 \%$ & $4.42 \%$ & $4.56 \%$ & $4.37 \%$ \\
\hline $\begin{array}{l}\text { Total qualified scientific, } \\
\text { therapeutic \& technical staff }\end{array}$ & $3.12 \%$ & $2.59 \%$ & $4.55 \%$ & $4.73 \%$ & $4.50 \%$ \\
\hline
\end{tabular}




\begin{tabular}{|l|l|l|l|l|l|}
\hline Qualified ambulance staff & $6.38 \%$ & $6.18 \%$ & $6.18 \%$ & $6.55 \%$ & $6.20 \%$ \\
\hline Support to clinical staff & $5.81 \%$ & $5.48 \%$ & $5.41 \%$ & $5.54 \%$ & $5.32 \%$ \\
\hline NHS infrastructure support & $3.92 \%$ & $3.68 \%$ & $3.66 \%$ & $3.75 \%$ & $3.58 \%$ \\
\hline $\begin{array}{l}\text { Other non-medical staff or those } \\
\text { with unknown classification }\end{array}$ & $1.19 \%$ & $1.10 \%$ & $1.03 \%$ & $2.61 \%$ & $1.27 \%$ \\
\hline
\end{tabular}

Source: Adapted from NHS Sickness Absence Rates, Annual Summary Tables, 2009-10 to 2013-14, Health and Social Care Information Centre, 2014 (Table 3) 